society-and, indeed was its founder-to protect and provide for patients who had been discharged recovered from la Salpêtrière and Bicêtre, as well as in another looking to the succor of the "fatherless children and widows" of deceased alienists, by organizing and presiding over «l'Association mutuelle des médecins aliénistes de France». His devotion to the patients of la Salpêtrière is an imperishable memory, and especially are his courage and self-denial, as shown during the epidemic of cholera in 1849, remembered at that institution.

A man of this stature, who made a fundamental contribution to neurophysiology, who did much to advance knowledge in the field of psychiatry, and who made a deep and lasting impression on the life of his generation deserves to be brought again to the attention of all those who are concerned with science and medicine and especially with psychiatry, for BAILLARGER's organic concepts are at long last coming into their own.

\title{
Wachsplastik und ihre Museen
}

\section{Von Edgar Goldschmid, Lausanne}

Vor etwa hundert Jahren sah sich JАсов GRImm bewogen, der zweiten Auflage seiner Geschichte der deutschen Sprache eine neue Vorrede mitzugeben, da ihm die vom Jahr 1848 nicht mehr recht zu den Zeitläuften von 1853 zu passen schien. Die Unruhen waren zu Ende, aber noch kein Frieden herrschte. Seine Beschäftigung mit Sprachgeschichte und seine «Gleichsetzung von Goten und Geten» schien nicht zeitgemäß. Was er nun hierzu verlautbart, glauben wir unserem Beitrag zur Festschrift für unseren Freund Sigerist zweckmäßig voranstellen zu dürfen.

Er sagt (p. VIII): «Jede wissenschaft hat ihre natürlichen grenzen, die aber selten dem auge so einfach vorliegen, wie das stromgebiet des bachs, in dessen mitte nach unseren weisthümern ein schneidendes schwert gesteckt wird, damit das wasser zu beiden seiten abfließe. willige forscher sollen also den verschlungenen pfaden folgen und bald leichteres bald schwereres geschühe anlegen, um sie betreten zu können. Wer nichts wagt gewinnt nichts und man darf mitten unter dem greifen nach der neuen frucht auch den mut des fehlens haben. aus dem dunkel bricht das licht hervor und der vorschreitende tag pflegt sich auf seine zehen zu stellen. von der großen 
heerstraße abwärts liebe ich es durch enge kornfelder zu wandeln und ein verkrochenes wiesenblümchen zu brechen, nach dem andere sich nicht niederbücken würden.»

Aus Wachs gegossene oder bossierte Gegenstände wie Perlen, Früchte, nackte Teile von Puppen und die sogenannten Friseursköpfe sind uns allen von klein auf geläufig. Kunstfreunde wissen die neapolitanischen Krippenfiguren zu schätzen, ein weiteres Publikum besucht die Wachsfigurenkabinette und besonders ihre «Schreckenskammern». Wissenschaftler kennen manchmal auch anatomische Wachsmodelle und -figuren; Techniker das Wachs im Metallguß.

Die Erfahrung sagt, man sieht, was man weiß; Dinge, die immer am gleichen Ort sichtbar sind, übersieht man aber leicht. Die Art der Darstellung eines Gegenstandes ändert sich mit der Erweiterung unserer Kenntnisse. Aber auch in der wissenschaftlichen Darstellung spielt die Mode eine Rolle; und das ist gar nicht verwunderlich, wenn man zum Vergleich das Auftauchen und Verschwinden bestimmter Sprachwendungen und Ausdrücke bedenkt.

Manchmal läßt sich ein Wiederauftauchen anscheinend vergessener Dinge oder Verfahren beobachten, ohne daß mit Sicherheit ein Grund oder eine Veranlassung deutlich würde.

In vielen naturwissenschaftlichen und anatomischen Instituten sind alte oder ältere Modelle und Figuren aus Wachs bossiert vorhanden, aus der Zeit zwischen etwa 1775 und 1830. Es handelt sich da weniger um einzelne Organe als um Organsysteme, Muskel-, Gefäß- und Nervenpräparation, ganze Glieder oder lebensgroße Figuren. Doch weder die embryologischen Modelle noch die dermatologischen Moulagen, deren Vogue vor etwa fünfzig Jahren einsetzte, haben sie zu neuem Leben erweckt. Ganz im Gegenteil erschienen sie - unansehnlich, verstaubt und vernachlässigt - nur wie arme Verwandte der neuen und frischlackierten Fabrikmodelle. Es gibt auch kleine, gerahmte Relieffiguren in Wachsguß (19. Jahrhundert) ganz in der Art, wie sie im 16. Jahrhundert, viel bewundert, von Italien nach Frankreich kamen.

So vergessen sie waren, glückte es doch bei systematischem und hartnäckigem Nachforschen in Instituten und Museen, in bescheidener Umgebung wie in pompösen staatlichen Instituten eine ganze Anzahl von Wachsbossierungen nachzuweisen und aufzudecken - selbst ganz wörtlich verstanden. 
Vor etwa sechzig Jahren wurden von den Kunsthandlungen Reproduktionen der «Jeune fille de Lille» angeboten, einer polychromen Wachsbüste der Sammlung Wigar (1762-1834) im Kunstmuseum in Lille. Der Grund hierfür ist uns nicht bekannt, keineswegs konnte es der gleiche sein wie der für die Überschwemmung des kunstfrohen Publikums mit der Porträtbüste der Nefre-Titi vor etwa dreißig Jahren.

Um so merkwürdiger erscheint eine solche einseitige Vorliebe und Vernachlässigung, wenn man bedenkt, daß zum mindesten zwei große Museen bestanden, in Wien und Florenz, zugänglich wie jedes andere Museum, die ganz ausschließlich anatomische, zoologische, chirurgische und pathologisch-anatomische sowie geburtshilfliche Wachsfiguren zur Schau bringen. (Besuchsverbot für weibliches Publikum haben wir nur in einem Pariser wissenschaftlichen Museum bemerkt.) Diese Museen bleiben leer, oder sie werden nur ganz ausnahmsweise - man möchte meinen: versehentlich aufgesucht. Das ist um so auffallender, als die Unternehmungen, die dem Publikum unter dem Namen Wachsfigurenkabinett bekannt sind, sich der allergrößten Beliebtheit erfreuen - auf dem Jahrmarkt im Dorf ebenso wie in den pompösen Ausstellungen der Großstadt. Obwohl alle Einzelheiten einer monographischen Schilderung vorbehalten bleiben müssen, sei hier daran erinnert, daß ein geduldiges Publikum in London vor «Madame Tussaud's Exhibition» (of Wax Works) stundenlang Schlange steht, daß in Paris das «Musée GrÉviN» alle Nachmittage und Abende von vollzähligen Familien überlaufen ist, wie auch "Castan's Panoptikum» in Berlin früher ein zahlreiches Publikum anzog.

Historisch interessierte Museumsbesucher oder Freunde der Kleinkunst kannten früher die in zahlreichen Sammlungen aufgestellten Pultvitrinen mit polychromen Wachsminiaturporträts, mit Perücken aus natürlichem Haar, mit Perlen und Edelsteinen, selbst mit Spitzen verziert, oft aus altem Familienbesitz. Uns fehlt die Erklärung für die Tatsache, daß diese höchst reizvollen und lehrreichen Kunstwerke entweder ganz aus der Ausstellung verschwunden oder in Schränken verwahrt sind, und daher dem Publikum unsichtbar bleiben, oder in völliger Dunkelheit ein kümmerliches Dasein fristen.

Wo solche Gegenstände in geringer Zahl - etwa ein Dutzend - noch sichtbar ausgestellt sind, fehlt der Hinweis, oder nähere Angaben sind überhaupt unbekannt, «Iliacos intra muros peccatur et extra». Auch lebensgroße Figuren aus Wachs oder wachsähnlichen Ersatzstoffen haben gelegentlich seltsame Schicksale erlitten: in der Kunstschule vom Hammer be- 
droht oder vom Gerichtsvollzieher beim Schaubudenbesitzer gepfändet, haben sie Zuflucht in naturwissenschaftlichen Museen gefunden. Einige sind da allerdings zu verdienten Ehren gelangt.

Die Herkunft aller dieser Wachsbossierungen verliert sich in alter Zeit. Wachs wurde immer gefunden, und künstlerische Begabung und Spieltrieb war immer vorhanden. Götterfiguren und Exvoto; Formen zum Metallguß sind nachweisbar; Zauberei an Wachsfiguren; Imagines und Wachsmasken sind bekannt; und erhalten ist auch die Verwendung von Wachsporträts nach Totenmasken, und solche gar als Einsatz in Terracottabüsten. Auch lebensgroße Wachsfiguren sind überliefert. Dazu kommen die «Agnus Dei» und die Wachssiegel. Wirkliche Kunstwerke sind früh bekannt, und ihre Herstellung wird von VASARI (1512-1574) genau beschrieben. Er erwähnt Andrea VerrocGhios (1432-1488) Einfluß auf die «Fallimagini» und zitiert besonders Orsino. Auf diesen geht auch eine moderne Datierung der «Jeune fille de Lille» zurück, die eine Zeitlang RAFFAEL zugeschrieben wurde. Adhuc sub iudice lis est.

Nun sind uns im Bargello drei Kunstwerke erhalten und genau datiert, interessanterweise von gleicher Bedeutung für die bildende Kunst wie für die Medizin: die prachtvolle Darstellung der Verwesung wie der Pest von der Hand des Giulio Gaetano Zumbo (1656-1701), von dem auch ein wachsbossierter Kopf in Florenz erhalten ist. Von hier aus laufen die Wege nach Bologna, Florenz, Paris und anderen Städten, wo sich dann Schulen der anatomischen Wachsbossierung entwickeln konnten. In der Porträtbossierung finden sich wohl überall einzelne Meister; zahlreiche Künstler werden in Italien, Frankreich und auch in Süddeutschland genannt. Die Kunst pflanzte sich fort seit der Hochrenaissance, besonders im 17. und im ganzen 18. Jahrhundert und (z. B. in Frankreich) bis in die Mitte des 19. Jahrhunderts.

Wie im großen, so im kleinen. Philipp Nathan Wilhelm Gurtius (1737-1791) in Bern begann als Arzt anatomische Wachsmodelle für sich zu bossieren, ging über zum Porträt und wurde dadurch (1761) an den französischen Hof gezogen. Er hatte Erfolg, stellte aus (an den Boulevards seit 1780; die Caverne des Grands Voleurs seit 1783), nahm seine Nichte Marie Grossholtz (1760-1850) aus Straßburg zu sich als Porträtistin, gründete ein Museum, wie es schon seit Beginn des 18. Jahrhunderts in Paris bestand - dieses ging zurück auf Antorne BenoIst (1632-1717), den «sculpteur de Cire du Roi», der von LouIs XIV. gefördert und geadelt worden war. Aber schon seit 1723 hatte DesnouËs anatomische Wachsmodelle in 
der Rue de Tournon ausgestellt. Um 1800 zog das Curtiussche Museum nach London, wo auch schon etwa seit der Mitte des 18. Jahrhunderts das Solomonsche Wachsfigurenmuseum bestand und z. B. 1772 vom berühmten Quäker-Arzt John FothergiLL (1712-1780) besucht wurde. Nach dem Gatten von Marie Grossholtz hieß das Museum bald und heute noch Mme Tussauds; aber die ursprünglichen anatomischen Figuren von CurTIUS' Hand sind dort verschollen. Auch bei Grévin sind solche nicht mehr beliebt; so daß der Schreiber dieser Zeilen dort mit einiger Herablassung an den Jardin des Plantes verwiesen wurde.

Sind die Folianten mit den Kupferstichen nach den Wachsfiguren im «Josephinum» von rein anatomischem Inhalt, so sind die englischen und französischen Bücher, die sich mit den populären «Wachsfiguren-Kabinetten» befassen, das von JoHn T. Tussaud wie das von JEAN GÉzAN, von historischem wie soziologischem Interesse, in Text und Bildern. Das gleiche wie für die Wachsfiguren gilt auch für die Wachsminiaturporträts, die sich in den verschiedenen Museen (und früheren Privatsammlungen) finden. So etwa die Familie Louis XIV. in Versailles, Mitglieder der Hofgesellschaft im Cluny oder den deutschen fürstlichen Sammlungen, berühmte Zeitgenossen im Carnavalet, der «Seckelmeister» im Berner Historischen Museum, die Gattin des Kupferstechers Hedlinger in Basel, Privatleute in Genf sowie der von Goethe geschilderte Joh. Chrn. SenkKeNBerg in Frankfurt oder wichtige Mitglieder der Bürgerschaft in Nürnberg.

Wenden wir uns nunmehr zu den ausschließlich medizinischen Sammlungen, so gehören zu den ältesten vielleicht die drei Figuren der Vatikanischen Bibliothek, die, von Maître JAGques D'Angoulème (geb. 1550) ausgeführt, dort aufbewahrt wurden: ein «Lebender», einer mit der Präparation von Muskeln, Gefäßen und Nerven, und ein Ecorché. Später findet sich die zunächst wohl wichtigste Sammlung in Bologna, der die meisten italienischen Universitäten folgen - Einzelheiten würden uns hier zu weit führen -, und sie wird endgültig von Florenz abgelöst. Unter Leitung von Felice Fontana (1730-1805) ist dort vom Bildhauer Clemente Susini (1754-1814) eine Art von Fabrik geführt worden, die nicht nur sozusagen alle Welt mit den schönsten anatomischen Wachsfiguren belieferte, sondern wo auch Wachsbossierer ausgebildet wurden. Diese führten nicht nur das Florentiner Unternehmen weiter, sondern sie haben, in ihre Heimat zu• rückgekehrt, dort selbständig weitergearbeitet. Von ihnen soll nur Petrus Koning (1787-1834) in Utrecht erwähnt werden, der das dort von JAN 
Bleuland (1756-1838) begründete Museum mit einer großen Zahl vorzüglicher Wachsbildwerke versah.

Auch Mascagni (1752-1815) und andere haben als Lehrmeister der Wachsbossierer gewirkt.

Die Florentiner Sammlung in der «Specola», die heute noch die zahlreichen Säle des Museums füllt, ist dem Großherzog LEopold (1747-1792) zu verdanken. Die berühmteste von allen, die von Kaiser Josef II. (1741 bis 1790) gestiftete Sammlung im Wiener Josephinum (1772), ist gerade wie die Florentiner Sammlung auch heute noch in der alten Gestalt und Schönheit dem Publikum zugänglich. Wer glaubt, daß diese Figuren nur eine Spielerei wären, kann sich im Genfer Naturwissenschaftlichen Museum belehren lassen, wie sehr die wenigen dort vorhandenen Figuren und «Präparate» von Lernbegierigen verlangt werden, die den anatomischen Universitätsunterricht aus irgendeinem Grund entbehren müssen. Auch in anderen Universitätsinstituten stehn lebensgroße Wachsfiguren zum Studium bereit.

Pathologisch-anatomische Präparate sind vielfach vorhanden - wir erwähnen hier nur die im Musée Dupuytren, im Museum des Royal College of Surgeons in Edinborough, in GuY's Hospital in London. Als Besonderheit sei erwähnt, daß die Edinburger Präparate von Sir Charles Bell (1774-1842), dem großen Physiologen, eigenhändig hergestellt wurden; die in GuY's Hospital von einem sonst nur wenig bekanntgewordenen Bildhauer, Joseph Towne (1808-1879).

Die an den verschiedenen Orten heute noch vorhandenen Exemplare sind natürlich von verschiedener Qualität, von ihrem meist guten Erhaltungszustand abgesehen. Aber auch ohne das Firmenschildchen ist die Diagnose «SusinI fecit» unschwer zu stellen.

Die Sammlungen dermatologisch-venerologischer Objekte sind zumeist um 1900 entstanden, mit modernen Moulageverfahren. Die berühmteste Sammlung im Pariser Hôpital St-Louis, der einstigen Wirkungsstätte des Baron Alibert (1766-1837), stammt wohl zumeist von Baretta, dem bekannten Mouleur und Diagnostiker (1849-1907), die früher sehr bekannte Sammlung der Lassarschen Klinik (1849-1907) in Berlin vom Präparator Kasten.

Besonders eindrucksvoll scheinen uns auch die geburtshilflichen - heutzutage etwas mißachteten! - Wachsfiguren der Florentiner Geburtshilflichen Klinik (von GaLletTi 1776), am schönsten immer die Bossierungen von Susini. Den vielerwähnten Florentiner «Lebbroso» von CaLAmaI 
haben wir leider nicht zu Gesicht bekommen, da er anscheinend zu gebrechlich ist, um seine Vitrine zu verlassen.

Eine große Anzahl von Wachspräparaten verschiedenen Charakters finden sich in Paris im Musée Orfilla und der Galerie d'Anatomie im Jardin des Plantes. Es ist kaum anzunehmen, daß sie dort noch ausgestellt wären, wenn sie nicht den Beweis ihrer Bedeutung für die Belehrung des Publikums erbracht hätten.

Vielleicht ist den medizinischen Wachsfiguren eine Auferstehung beschieden, wenn sich die anatomischen und pathologisch-anatomischen Institute durch die Überfüllung ihrer Präparier- und Seziersäle gezwungen sehen, auf diese Reserve zurückzugreifen.

Die Frage, ob wir ein Recht hatten, diese Untersuchung unserem Jubilar als Festgabe darzubringen, glauben wir damit beantworten zu können, daß vor drei Jahren noch ein «Unsterblicher», M. Edmond JALoux von der Académie Française, seinen vielgelesenen Roman Les Figures de Cire dem Problem der Einwirkung des Wachsfigurenkabinettes auf die Psyche der Beschauer gewidmet hat.

Für Einzelheiten, Literatur und Bilder, müssen wir auf die in Vorbereitung befindliche Monographie verweisen 